\title{
A SIMPLE MODEL OF THE DERIVATION OF FLUID MECHANICS FROM THE BOLTZMANN EQUATION'
}

\author{
BY H. P. McKEAN, JR.
}

1. Introduction. Numerous problems arising from nature can be described by a (possibly nonlinear) parabolic equation of the form

$$
\begin{gathered}
\partial p / \partial t=Q[p] \quad(t>0), \\
\lim _{t \downarrow 0} p=f .
\end{gathered}
$$

$Q$ is independent of $t>0$ and maps a function $f$ belonging to a certain manifold $M$ into a tangent vector $Q[f]$ based at $f$, so that the flow defined by (1) is a flow on $M$.

Now it may happen that the solutions of (1) come rapidly close to a special submanifold $H$ in which a striking simplification takes place: namely, the amount of information needed to distinguish points of $H$ is suddenly much smaller than in the ambient manifold $M$, and owing to this simplification of the function space, the flow defined by (1) can be described much more simply. This phenomenon is familiar to students of statistical mechanics, notably in the passage pass from Boltzmann's equation to hydrodynamics via the ChapmanEnskog expansion; see, for instance, Ford-Uhlenbeck [2].

I will speak about 3 examples of this state of affairs. The first is due to Carleman [1, p. 106]. He attaches no particular significance to it, but it is cute and illustrates some of the ideas involved. The second example is the actual Chapman-Enskog-Hilbert development for the Boltzmann equation, or at least a conjecture as to how it should go; see $\$ 3$ below. Boltzmann's equation is too complicated to prove very much about, but in the simplified model of $\$ 4$ it is possible to compute everything and to see explicitly all the phenomena that the Boltzmann equation is supposed to exhibit. ${ }^{2}$ Grad [3] discussed the Chapman-Enskog-Hilbert development for the linear approximation to the Boltzmann equation. This is still very complicated, so naturally the results are less satisfactory.

2. Carleman's example. Carleman, in his study of the Boltzmann equation [1], used the problem

${ }^{1}$ An address delivered before the New York meeting of the Society by invitation of the Committee to Select Hour Speakers for Eastern Sectional Meetings, April 12, 1968; received by the editors September 20, 1968.

2 This material is taken from McKean [6]. 


$$
\ddot{x}=(\dot{x})^{2}-x^{2}
$$

to illustrate the ideas involved in the Chapman-Enskog-Hilbert development.

Replace (1) by the coupled pair

$$
\left(\begin{array}{l}
\dot{x} \\
\dot{y}
\end{array}\right)=\left(\begin{array}{c}
y \\
y^{2}-x^{2}
\end{array}\right)=Q
$$

and let $M=R^{2}$ be the associated phase plane. The special submanifold $H$ is now singled out by means of the following curious recipe: put an extra factor $1 / \epsilon$ in front of $Q$ and ask that the solution be a (formal) power series in $\epsilon$; then put $\epsilon$ back $=1$ and hope for convergence to a bona fide solution of (2). This is plainly a very radical thing to do: $\epsilon$ is put in upside down and comes out right-side up, esp., to avoid poles and worse, the coefficients of this (formal) power series must satisfy a whole string of identities whose significance is not at all apparent! Naturally, (2) may not have any solutions of this type.

Hope for the best, insert the $1 / \epsilon$ into (2), and ask for a formal power series solution $x=x_{0}+\epsilon x_{1}+\cdots$. This gives

$$
\ddot{x}_{n-1}=\sum_{i+j=n}\left[\dot{x}_{i} \dot{x}_{j}-x_{i} x_{j}\right] \quad(n \geqq 0)
$$

with the understanding that $x_{-1} \equiv 0$. (3) can be solved step-wise, beginning with $\ddot{x}_{-1}=0=\left(\dot{x}_{0}\right)^{2}-x_{0}^{2}$. The only reasonable solution is $x_{0}=$ constant $\times \exp ( \pm t)$, and you can go on step by step. Carleman pointed out an easier way. Under the substitution $x \rightarrow \epsilon a$ and $(\dot{x})^{2} \rightarrow \epsilon^{2} b$, the problem becomes

$$
b={ }_{2} \dot{a}\left(b-a^{2}\right),
$$

and it is easy to guess a first integral of this:

$$
b=a^{2}+a+1 / 2 .
$$

Now $x$ is supposed to be a formal power series, so $a$ and $b$ should be formal Laurent series:

(6) $a=a_{-1} / \epsilon+a_{0}+\cdots, \quad b=b_{-2} / \epsilon^{2}+b_{-1} / \epsilon+b_{0}+\cdots$.

(5) is consistent with this, and it is not difficult to check that (5) is the only possible first integral in the domain of formal Laurent series. Going back to $x$ and $\dot{x}$ and putting $\epsilon=1$, one finds the special first integral

$$
(\dot{x})^{2}=x^{2}+x+1 / 2=(x+1 / 2)^{2}+1 / 4 .
$$


This corresponds to the 2 dark hyperbolas, labelled $k=0$ in the dia-

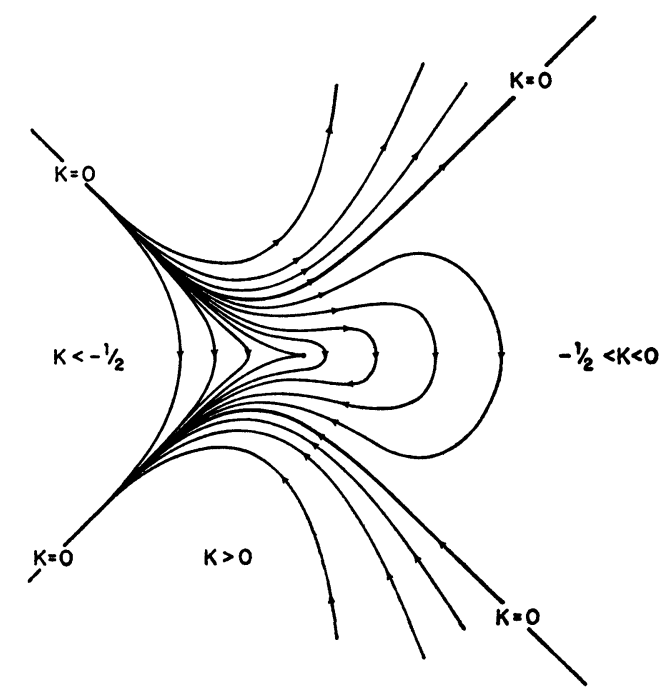

gram, comprising the desired submanifold $H$. The other solutions may be found from the general first integral:

$$
(\dot{x})^{2}=k e^{2 x}+x^{2}+x+1 / 2 \quad(-\infty<k<\infty),
$$

and one notices with satisfaction that every solution approaches $H$ either for $t \uparrow \infty$ or for $t \downarrow-\infty$. (1) is replaced in $H$ by the simpler problem

$$
\dot{x}= \pm\left((x+1 / 2)^{2}+1 / 4\right)^{1 / 2}
$$

which can be solved explicitly. As an unexpected bonus, you find that $H$ separates the solutions which cross the real line from those which do not. I do not want to attribute any deep significance to this example, but you see that the power series trick has singled out a particularly nice solution!

3. Boltzmann to hydrodynamics via the Chapman-Enskog expansion. Boltzmann's equation

$$
\frac{\partial p}{\partial t}+v \cdot \frac{\partial p}{\partial x}+f \cdot \frac{\partial p}{\partial v}=B[p \otimes p]
$$

is supposed to govern the distribution $p d v$ of the velocity $v \in R^{3}$ of a typical molecule in its dependence upon time $t \geqq 0$ and position $x \in R^{8}$. 
The left-hand side is a streaming term. $\partial / \partial x$ is the spatial gradient, $\partial / \partial v$ is the velocity gradient, and $\mathfrak{f}$ is the external field. $B=B[p \otimes p]$ accounts for the change due to collisions between molecules. It is of degree 2 in $p$ as the notation suggests, though its precise expression need not bother us. Hilbert [4] proposed to identify fluid mechanics with the (formal) power series solutions of

$$
\frac{\partial p}{\partial t}+v \cdot \frac{\partial p}{\partial x}+\mathfrak{f} \cdot \frac{\partial p}{\partial v}=\epsilon^{-1} B[p \otimes p],
$$

putting $\epsilon$ back $=1$ and hoping for convergence. A rough idea of what he had in mind can be obtained as follows.

Application of the recipe to the formal power series solution $p=p_{0}+\epsilon p_{1}+\cdots$ leads at once to the rule

$$
\left(\frac{\partial}{\partial t}+v \cdot \frac{\partial}{\partial x}+\mathfrak{f} \cdot \frac{\partial}{\partial v}\right) p_{n-1}=\sum_{i+j=n} B\left[p_{i} \otimes p_{j}\right] \quad(n \geqq 0)
$$

with the understanding that $p_{-1} \equiv 0$. At the stage $n=0$, (3) says that $0=B\left[p_{0} \otimes p_{0}\right]$. This happens only if $p_{0}$ is a local Maxwellian distribution, meaning that $p_{0}=c_{0} \exp \left(-c_{2}\left|v-c_{1}\right|^{2}\right) .\left(c_{0}, c_{1}, c_{2}\right) \in R^{5}$ is the hydrodynamical state. It depends upon the 5 moments $\int v^{n} p_{0} d v(n=0$, $1,2)$ only and is a function of $(t, x) \in[0, \infty) \times R^{3}$. The local Maxwellian form signifies a (local) steady state as regards collisions, and the formal power series $p$ is an expansion around this state.

At the next stage, you begin to get some useful information. (3) can be expressed as

$$
\left(\frac{\partial}{\partial t}+v \cdot \frac{\partial}{\partial x}+\mathfrak{f} \cdot \frac{\partial}{\partial v}\right) p_{n-1}-\sum_{i, j \neq 0, n} B\left[p_{i} \otimes p_{j}\right]=C\left[p_{n}\right],
$$

in which $C[f]=B\left[p_{0} \otimes f\right]+B\left[f \otimes p_{0}\right]$ is a nice selfadjoint integral operator acting in an appropriate Hilbert space. Given $p_{0}, \cdots, p_{n-1}$, this is an integral equation for $p_{n}$, and to solve it you have to obey the dictates of the Fredholm alternative: you must make the left-side perpendicular to the null-space of $C$. Now the null-space of $C$ is very simple. It is just the 5 -dimensional subspace spanned by $v^{n} p_{0}(n$ $=0,1,2)$, and if you make the left side of (4) perpendicular to it, you will get some new information about $p_{0}, \cdots, p_{n-1}$. At the stage $n=1$, this is information about the hydrodynamical state of $p_{0}$, and if you interpret this state in terms of density, fluid velocity, and temperature, you get the Eulerian hydrodynamical equations.

Now having satisfied the Eulerian equations, you can go on and solve (4) with $n=1$ for $p_{1}$. You will not get the whole of $p_{1}$, only the 
part that is perpendicular to the null-space of $C$, so you still have a 5 -dimensional piece to specify, and that is done by imposing the Fredholm alternative at the next stage $(n=2)$. This gives information on the hydrodynamical state of the next approximation $p_{0}+\epsilon p_{1}$, and what you find is the Navier-Stokes equations which constitute the conventional basis for viscous fluid mechanics. Now you solve (4) for the part of $p_{2}$ which is perpendicular to the null-space of $C$ and impose the Fredholm alternative at the next stage $(n=3)$, obtaining equations for the hydrodynamical state of $p_{0}+\epsilon p_{1}+\epsilon^{2} p_{2}$; these turn out to be a big mess. Eventually, you get the whole formal power series $p=p_{0}+\epsilon p_{1}+\cdots$ and what may be called the correct hydrodynamical equations. Putting $\epsilon$ back $=1$, you hope that everything will converge and single out a nice submanifold $H$ inside the manifold $M$. On the hydrodynamical equations define the same flow as the (more complicated) Boltzmann equation does. This submanifold is distinguished by the fact that $f \in H$ can be recovered from its 5 hydrodynamical moments $\int v^{n} f d v(n=0,1,2)$, so that to specify a solution curve in $H$ you have only to give these moments at time $t=0$. This is what Ford-Uhlenbeck [2] call the Hilbert paradox. The (very complicated) formula which expresses $f \in H$ in terms of its 5 moments is the so-called Chapman-Enskog expansion.

The situation is recapitulated in the lower half of the accompanying diagram. This half of the diagram is commutative. The upper half has to do with the approximation of the general solution $p_{*}$ of the

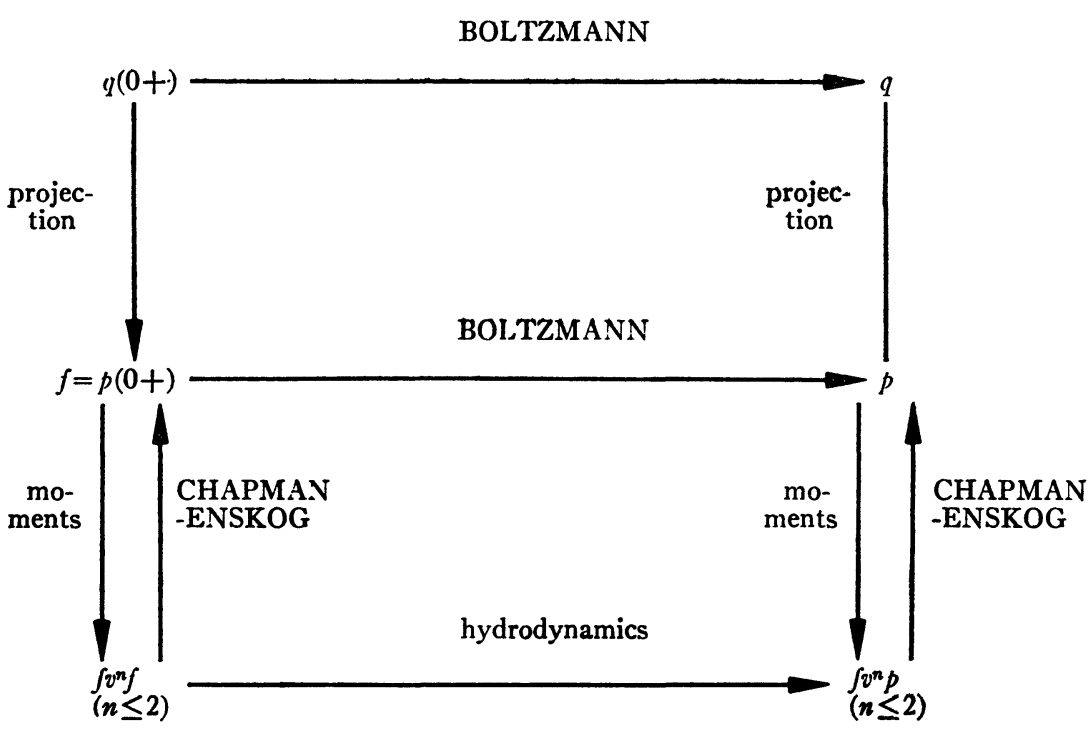


Boltzmann equation by one of these hydrodynamical solutions $p$. I am not sure how widely this next statement will be believed among physicists, but it is correct in simplified models, and hopefully so in general, that there is (a nonlinear) projection onto the submanifold $H$ which commutes with the Boltzmann flow, as indicated in the diagram, such that the general solution $p_{*}$ and its projection $p$ come rapidly close to one another. Consequently, for all practical purposes, $p_{*}$ can be replaced first by $p$ and then by $\int v^{n} p d v(n=0,1,2)$ which is a much simpler affair. This is what Ford-Uhlenbeck [2] call a contraction of the description.

Boltzmann's equation is too complicated to permit us to check all this at the present stage of the mathematical art, so it is comforting to see all the conjectured behavior appearing explicitly in the simplified model of the next section.

4. A simpler model. ${ }^{3}$ The simplified model cited above is based on

$$
\partial p / \partial t+e \partial p / \partial x=p(-e)-p(+e)=D[p] .
$$

Here $e$, which is a velocity, is simply $\pm 1, x$ runs over $R^{1}$ instead of $R^{3}$, the external field $f$ is absent, and the collision term is merely linear. Naturally, $p$ is a function of $(t, x, e) \in[0, \infty) \times R^{1} \times( \pm 1)$, but mostly I will write $p=p(e)$ as in (1). This problem governs the distribution of a particle moving on the line according to the rule $\dot{x}= \pm 1$ with independent exponential holding times between changes of velocity [ $\pm 1 \rightarrow \mp 1]$; see $\mathrm{Kac}[5]$. Using this model, it is easy to write down the solution of (1) and to check that if $f=\lim _{t \downarrow 0} p$ belongs to $C^{\infty}\left[R^{1} \times( \pm 1)\right]$, then $p \in C^{\infty}\left[[0, \infty) \times R^{1} \times( \pm 1)\right]$; naturally, the solution is positive if $f$ is positive. (1) is connected with the telegraph equation, and it will be important to know this later on. To see this, you compute $\partial^{2} p / \partial t^{2}$ from (1) and obtain

$$
\partial^{2} p / \partial t^{2}+2 \partial p / \partial t=\partial^{2} p / \partial x^{2} .
$$

The important feature of (2) is that $e$ has dropped out! But a price is paid for this in that the initial data has to be augmented by the knowledge of

$$
\lim _{t \downarrow 0} \partial p / \partial t=-e f^{\prime}+D[f]
$$

I am going to play Hilbert's trick on this, looking for formal power series solutions of

$$
\partial p / \partial t+e \partial p / \partial x=\epsilon^{-1} D[p],
$$

McKean [6] gives full proofs of all the statements in this section. 
putting $\epsilon$ back $=1$ and hoping for the best. Put the formal power series $p=p_{0}+\epsilon p_{1}+\cdots$ into (4) and match like powers. This gives

$$
(\partial / \partial t+e \partial / \partial x) p_{n-1}=D\left[p_{n}\right] \quad(n \geqq 0)
$$

with the usual understanding that $p_{-1} \equiv 0$. The first thing you notice is that $D\left[p_{0}\right]=p_{0}(-e)-p_{0}(+e)=0$, which says that $p_{0}$ is an even function of $e$, i.e., it is independent of $e$. Now at the next stage $(n=1)$, the dictates of the Fredholm alternative must be satisfied. The nullspace of $D$ is simply the even functions of $e$, so the left side of (5) $\left[(\partial / \partial t+e \partial / \partial x) p_{0}\right]$ must be odd. But $p_{0}$ is even, so you must have

$$
\partial p_{0} / \partial t=0,
$$

i.e., $p_{0}$ is independent of $t \geqq 0$ also. This fact, trivial as it may be, is the analogue of the Eulerian equations. (5) can now be solved for the odd part of $p_{1}$. Then you go to (5) for $n=2$ and impose the Fredholm alternative which says that $(\partial / \partial t+e \partial / \partial x) p_{1}$ must be odd. This gives the whole of $p_{1}$,

$$
\left(p_{1}\right)_{\text {odd }}=-(e / 2) f_{0}, \quad\left(p_{1}\right)_{\text {even }}=\left(f_{1}\right)_{\text {even }}+(1 / 2) t f_{0}^{\prime \prime},
$$

and you should be able to see in this some counterpart to the NavierStokes equations. The analogue of the hydrodynamical state is just $p(-1)+p(+1)$, so you have to find some equation for $\left(p_{0}+\epsilon p_{1}\right)(-1)$ $+\left(p_{0}+\epsilon p_{1}\right)(+1)$ which is valid modulo $\epsilon^{2}$. This is simply the heat equation, as is plain from (6), (7), and

$$
\left(\frac{\partial}{\partial t}-\frac{\epsilon}{2} \frac{\partial^{2}}{\partial x^{2}}\right)\left(p_{0}+\epsilon p_{1}\right)=\frac{\epsilon \partial p_{1}}{\partial t}-\frac{\epsilon}{2} \frac{\partial^{2} p_{0}}{\partial x^{2}}+\text { terms in } \epsilon^{2} .
$$

To go beyond this point, the use of (5) is inefficient, so I shall simply state the facts and prove them by a better method based on the telegraph equation (2).

TheOREM. Given a formal power series $f=f_{0}+\epsilon f_{1}+\ldots$ with coefficients from $C^{\infty}\left[R^{1} \times( \pm 1)\right]$, (4) admits a formal power series solution $p=p_{0}+\epsilon p_{1}+\cdots$ with coefficients from $C^{\infty}\left[[0, \infty) \times R^{1} \times( \pm 1)\right]$ which reduces to $f$ at $t=0+$ if and only if

$$
-e(f)_{\text {odd }}=\left[\left(1+\epsilon^{2} \partial^{2}\right)^{1 / 2}-1\right](\epsilon \partial)^{-1}(f)_{e v e n} ;
$$

- $\partial$ stands for spatial differentiation, and the radical is to be expanded according to the binomial formula. 
moreover, $(p)_{\text {odd }}$ and $(p)_{\text {even }}$ are related in the same manner at each later time $t \geqq 0$, and

$$
\frac{\partial p}{\partial t}=\epsilon^{-1}\left[\left(1+\epsilon^{2} \partial^{2}\right)^{1 / 2}-1\right] p
$$

in addition to (4), esp.,

$$
\frac{\partial}{\partial t}(p)_{e v e n}=\epsilon^{-1}\left[\left(1+\epsilon^{2} \partial^{2}\right)^{1 / 2}-1\right](p)_{e v e n} .
$$

REMARK. $2(p)_{\text {even }}$ is the hydrodynamical state. Hilbert's paradox is seen in the fact that the even part of $f$ determines the whole solution. (9) is the Chapman-Enskog expansion, defining the hydrodynamical submanifold $H$. (11) is the full hydrodynamical equation; the right-hand side of (11) reduces to $(\epsilon / 2) \partial^{2} / \partial x^{2}$ in the second approximation, as it should.

PROOF. The principal trick is to notice that the telegraph equation associated with (4) $\left[\partial^{2} p / \partial t^{2}+(2 / \epsilon) \partial p / \partial t=\partial^{2} p / \partial x^{2}\right]$ can be factored:

$$
\left(\frac{\partial}{\partial t}+\frac{1+\left(1+\epsilon^{2} \partial^{2}\right)^{1 / 2}}{\epsilon \partial}\right)\left(\frac{\partial}{\partial t}+\frac{1-\left(1+\epsilon^{2} \partial^{2}\right)^{1 / 2}}{\epsilon \partial}\right) p=0 .
$$

The second factor maps the formal power series $p$ into a new formal power series, while the only formal power series that the first factor annihilates is 0 itself. This proves (10), and (9) follows by comparing the expressions for $\partial p / \partial t$ at $t=0$ obtained from (10) and from (4). The rest is trivial.

The next topic is the question of convergence; for this part it is natural to take $(f)_{\text {even }}=f_{0}$ (i.e., to drop the higher powers) since one puts $\epsilon=1$ eventually. The formal power series $(f)_{\text {odd }}$ is now computed from (9), and you find that it converges for $|\epsilon|<1$ iff $f_{0}$ admits an integral extension into the complex plane which is of exponential type $\leqq 1$; moreover, $p$ follows suit if this condition is satisfied, i.e., it also converges for $|\epsilon|<1$. This point is to be emphasized: to obtain convergence of the formal power series, the initial datum has to be very smeoth.

I now impose an additional condition just to simplify life: $\left\|(f)_{\text {even }}\right\|_{1}<\infty$. This is not unnatural since, if $(f)_{e v e n}$ is positive, this integral is the total amount of "fluid." Because $(f)_{\text {even }}$ is also of exponential type $\leqq 1$, its Fourier transform vanishes outside $[-1,+1]$, so that

$$
(f)_{\text {even }}=\int_{-1}^{+1} \exp (i \gamma x)(f)_{\text {evend }} d \gamma
$$


and

$$
(f)_{\text {odd }}=\int_{-1}^{+1} \exp (i \gamma x)(-e / i \gamma \epsilon)\left[\left(1-\epsilon^{2} \gamma^{2}\right)^{1 / 2}-1\right](f)_{\text {oven }}^{\hat{a}} d \gamma
$$

by (9), esp., you see from (13b) that ( $f)_{\text {odd }}$ is actually continuous on the closed disc $|\epsilon| \leqq 1$. $f$ itself can now be computed and a formula for $p$ deduced from (10):

$$
p=\int_{-1}^{+1} \exp (i \gamma x) \exp \left[t\left(1-\epsilon^{2} \gamma^{2}\right)^{1 / 2}-1 / \epsilon\right] \hat{f} d \gamma
$$

This is a bona fide infinitely differentiable solution of (4) even for $\epsilon=1$.

Now put $\epsilon=1$ and let us take up the question of the degree of approximation of the general solution $p_{*}$ of (1) by such special hydrodynamical solutions. Pick the initial datum $f_{*}$ of $p_{*}$ from $L^{1}\left[R^{1} \times( \pm 1)\right]$ and map it into a new function $f$ by the rule

$$
\begin{aligned}
\hat{f} & =\frac{1}{2}\left[1+\frac{1+D-i \gamma e}{\left(1-\gamma^{2}\right)^{1 / 2}}\right] \hat{f}_{*} & & \text { for }|\gamma| \leqq 1, \\
& =0 & & \text { for }|\gamma|>1 .
\end{aligned}
$$

This mapping is a projection, it commutes with solving (1), $f$ satisfies (9) (i.e., the mapping is projection onto $H$ ), and the corresponding solution $p$ of (1), given by (14) with $\epsilon=1$, differs from $p_{*}$ by a transient that washes out like $e^{-t}$, all as it should be. The proof is not at all hard, and I will not bother you with it.

A final point I want you to notice is that the physical picture demands that $p$ be positive. (10) preserves this property inside $H$ but not outside. This is because (10) is the same as (1) inside $H$ but not outside.

5. Carleman's model. The problem (1) of $\$ 4$ has the undesirable property that the collision term is linear. Carleman $[1$, p. 104] proposed an allied model that is a little closer to the Boltzmann equation:

$$
\partial p / \partial t+e \partial p / \partial x=D\left[p^{2}\right]
$$

for which you can imitate part of the formal power series stuff of $\$ 4$. A Chapman-Enskog expansion

$$
-e\left(f_{\text {odd }}\right)=E\left[(f)_{\text {even }}\right]
$$

exists, $E$ being a mapping of formal power series, and this expansion commutes with the flow induced by (1), so that you can substitute $-e(p)_{\text {odd }}=E\left[(p)_{\text {even }}\right]$ into $(1)$ and see what happens. This gives 


$$
\left(1-d E_{f}\right)^{2}\left[f^{\prime}\right]=\epsilon^{-1} f \times E[f]
$$

for any even formal power series $f, d E_{f}$ being the (formal) differential of $E$ at the place $f$. Unfortunately, $\left(1-d E_{f}\right)^{2}$ is evaluated only in the special direction $f^{\prime}$, so (3) is probably not easy to solve; in any case, it seems to be a novel kind of problem. The question of the convergence of such expansions is wide open.

Note added during the editing. A few additional (linear) models, such as the 3-dimensional Lorentz and Krook models, have recently been treated along the lines of $\$ 4$ by $E$. Hauge, J. van Leeuven, and myself [unpublished]; in fact, with this additional experience, the linear approximation to the Boltzmann equation now seems to be within the realm of possibility, but this is not yet done, and the difficulties look formidable.

\section{REFERENCES}

1. T. Carleman, Problemes mathematiques dans la theorie cinetique des gas, Almquist and Wiksell, Uppsala, 1957.

2. G. W. Ford and G. E. Uhlenbeck, Lectures in statistical mechanics, Lectures in Appl. Math., vol. 1, Amer. Math. Soc., Providence, R. I., 1963.

3. H. Grad, Asymptotic theory of the Boltzmann equation, Phys. Fluids 6 (1963), 147-181.

4. D. Hilbert, Grundzilge einer abgemeinen Theorie der linearen Integralgleichungen, Chelsea, New York, 1953. 1959.

5. M. Kac, Probability and related topics in the physical sciences, Wiley, New York,

6. H. P. McKean, Jr., Chapman-Enskog-Hilbert expansion for a class of solutions of the telegraph equation, J. Math. Phys. 8 (1967), 547-552.

Mombasa: July 8, 1968. 